\title{
Group-based intervention of participants with Parkinson disease: Findings from a 6-month LOUD Crowd ${ }^{\circledR}$ program
}

Sabiha Parveen

Department of Communication Sciences and Disorders, Oklahoma State University, Stillwater, USA

Purpose: Approximately 70-80\% of individuals with Parkinson disease (PD) experience some change in voice, and about 55\% experience changes in articulation with disease progression. Although an extensive literature exists about the benefits of well-known treatment programs such as LSVT LOUD ${ }^{\circledR}$ and LSVT BIG ${ }^{\circledR}$, limited studies are currently available that shed light on possible benefits of relatively new programs called SPEAK OUT! ${ }^{\circledR}$ and LOUD Crowd $^{\circledR}$ across both speech and non-speech dimensions among participants with PD. Therefore, the current study examined the possible benefits of a group-based LOUD Crowd ${ }^{\circledR}$ program among participants with PD across multiple speech and non-speech domains (i.e., voice, swallowing, cognition, self-reported depression, and VRQOL) during six months.

Methods: Participants included six individuals with PD in the treatment (TX) group who participated in a weekly hour-long LOUD Crowd ${ }^{\circledR}$ program. In contrast, the control (CN) group included three individuals with PD who did not receive any speech or cognitive treatment specific to their PD symptoms before or during the study. All participants were within mildmoderate severities of hypokinetic dysarthrias.

Results: No statistically significant group differences were observed between the TX and the $\mathrm{CN}$ groups at the end of six months. However, descriptive analyses suggested that the TX group demonstrated improved performance for different areas, including the vowel space, live loudness levels, cognitive performance, swallowing abilities, and VRQOL scores compared to the CN group.

Conclusions: The current study suggests possible benefits of a group-based LOUD Crowd ${ }^{\circledR}$ program among participants with PD for a variety of speech and non-speech areas of functioning.

Keywords: Group intervention, Parkinson disease, LOUD Crowd ${ }^{\circledR}$, Voice, Cognition, Swallowing, VRQOL

\section{INTRODUCTION}

Parkinson disease (PD) is a progressive neurological disorder typically associated with hypokinetic dysarthria and linked to abnormalities of respiration, phonation, articulation, resonance, and prosody [1]. It is the second most common degenerative disorder affecting people over the age of 65. Approximately 10 million worldwide are currently living with PD [2]. Existing research indicates about $70-80 \%$ of individuals with PD experience some change in voice, and about 55\% experience changes in articulation with disease progression [3]. Speech and voice changes are often the first sign to an individ-

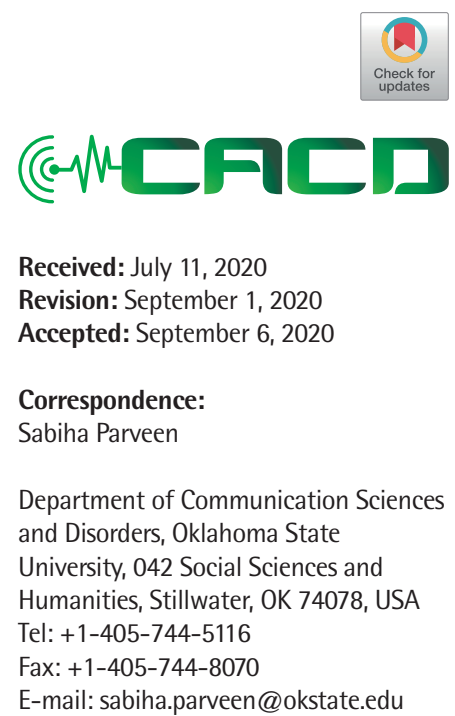

(C) 2020 The Korean Association of SpeechLanguage Pathologists

This is an Open Access article distributed under the terms of the Creative Commons Attribution NonCommercial License (https://creativecommons.org/ licenses/by-nc/4.0/) which permits unrestricted noncommercial use, distribution, and reproduction in any medium, provided the original work is properly cited. 
ual or family that something has changed before the clinical diagnosis. Thus, it is important to develop effective speech programs to improve the communicative effectiveness of individuals with PD. De Angelis and his colleagues examined the effectiveness of physiological speech exercise on a groupbased vocal rehabilitation program among 20 participants with PD with mild to moderate disease severity [4]. The authors specifically discussed the positive impact of group therapy in terms of increasing the motivation levels as well as the exchange of experiences among participants with PD and between the communication partners. The authors suggested the need for additional long-term studies to more specifically examine the benefits and maintenance of group-based rehabilitation programs for individuals with PD.

During the past two decades, several studies related to Lee Silverman Voice Treatment (LSVT ${ }^{\circledR}$ ) and its different modifications (i.e., LSVT ${ }^{\circledR}$ Clear, LSVT ${ }^{\circledR}$ LOUD, LSVT $^{\circledR}$ BIG), music therapy, and traditional therapy have been published. The LSVT LOUD $^{\circledR}$ program consists of a single target and cueing strategy of "think loud." The main premise of the program is to facilitate neuromotor adaptation during speech production such that the elevated effort level and increased amplitude of motoric activity become automatic and more accurately selfmonitored by the individual with PD with practice [1]. Different modifications of the program have been developed and reported by several researchers during the past two decades. Another vocal scaling program aimed for individuals with PD called the SPEAK OUT! ${ }^{\circledR}$ and LOUD Crowd ${ }^{\circledR}$ was developed in 2010. These programs have goals of improved functional communicative ability by speaking with intent as a vehicle [5]. The intent in this program is typically defined and modeled as a purposeful cognitive focus related to increased vocal loudness and intonation variability during speech tasks [6]. The first phase of the program, called SPEAK OUT! ${ }^{\circledR}$, involves 12 individual sessions. Once the participant completes the individual sessions, he/she transitions to a weekly maintenance program called LOUD Crowd ${ }^{\circledR}$. Each session of the SPEAK OUT! ${ }^{\circledR}$ and LOUD Crowd ${ }^{\circledR}$ is structured with a hierarchy of speech, voice, and cognitive exercises. The session includes warm-up vocalizations, sustained vowel phonation, pitch glides, counting, reading, and structured cognitive activities [1]. Similar to the LSVT LOUD ${ }^{\circledR}$ program, the SPEAK OUT! ${ }^{\circledR}$ program includes a home practice component to facilitate the use of strategies outside of the therapy session. The following sections summarize some of the key findings from the existing treatment programs for individuals with PD.

\section{Prior studies related to the effects of LSVT ${ }^{\circledR}$ program}

Spielman and his colleagues examined pre and post-treatment effects LSVT ${ }^{\circledR}$ on vocal loudness among 12 individuals with PD during two weeks [7]. Results indicated a significant increase in loudness for different speech tasks from pre- to post-treatment, a significant increase from pre- to follow-up phase. However, there was no significant loudness ratings between post-treatment and the follow-up phase. In addition, the VHI ratings were not significantly different among the participants' pre and post LSVT $^{\circledR}$ treatment. Similar benefits of a modified LSVT ${ }^{\mathbb{B}}$ program on phonatory performance (including vocal loudness, F0 range, and maximum phonation time) were reported by a prior study from 2011 involving 15 participants with PD during an 8-week weekly program [8]. Contrary to Spielman and his colleagues' findings, the 2011 study [8] reported significantly better VHI scores among the participants following the LSVT ${ }^{\circledR}$ program. Both studies discussed some of their limitations, including the lack of a control group, a limited number of participants and a lack of observation at different follow-up times post completion of LSVT ${ }^{\circledR}$.

More recently, Edwards, Theodoros, and Davidson examined the effectiveness of a group-based therapy (LOUD and PROUD, a modified version of LSVT ${ }^{\circledR}$ LOUD) among 13 individuals with PD during an eight week 90-minute weekly sessions (once a week for eight weeks) [9]. Results indicated a statistically significant increase in loudness (SPL level) for sustained vowel production, reading, monologue, and conversation following 8-weeks of group therapy. However, there were no significant differences for maximum frequency range, duration of sustained vowels, and other self-reported communication measures pre and post-therapy. The authors suggested careful interpretation of the findings due to the lack of statistically significant differences, small sample size, and a high degree of variability in treatment response. The authors also concluded the need for future studies examining the impact of group therapy among PD participants in terms of loudness (SPL) and other factors such as timing, frequency, and dosage of intervention for optimal maintenance.

\section{Studies related to effects of SPEAK OUT! ${ }^{\circledR}$ and LOUD Crowd ${ }^{\circledR}$}

To the best of our knowledge, there are five known published reports about possible effects of SPEAK OUT! ${ }^{\circledR}$ and LOUD Crowd $^{\circledR}$ programs among participants with PD. The first study reporting the SPEAK OUT! ${ }^{\circledR}$ program's effectiveness was published in 2014 [10]. The study examined six individuals with PD during 12 weeks and reported improvements in vocal in- 
tensity for vowels during structured speech tasks and self-reported voice-related quality of life (VRQOL) performance at the end of treatment. Similar findings of improvements in vocal intensity for both sustained vowels and during a conversational speech following completion of the SPEAK OUT! ${ }^{\circledR}$ program were reported in a different study in 2015 [11]. This study included 11 participants with PD who completed 12 individual SPEAK OUT! ${ }^{\circledR}$ sessions for four weeks. Specifically, the authors reported a mean increase of $8 \mathrm{~dB}$ SPL in sustained /a/ production and a $7 \mathrm{~dB}$ increase in SPL during a conversational speech at the end of four weeks. The authors concluded the SPEAK OUT! ${ }^{\circledR}$ program's effectiveness in improving the vocal loudness of participants with PD.

In a separate study, Levitt, Chitnis, and Walker-Batson examined the voice performance of 12 individuals with PD during 12 weeks [12]. This study included measurements before treatment (baseline, T1), completion of individual phase (SPEAK OUT! ${ }^{\circledR}$; T2), after attending four sessions of group therapy (LOUD Crowd ${ }^{\circledR}, T 3$ ), and eight sessions of group therapy (LOUD Crowd $\left.{ }^{\circledR}, \mathrm{T} 4\right)$. Results indicated a significant increase in vocal loudness since initiation of treatment (at all three-time points) compared to baseline (T1). In addition, participants reported a significant improvement in VRQOL scores following treatment as compared to the baseline. The authors concluded that the SPEAK OUT! ${ }^{\circledR}$ and LOUD Crowd ${ }^{\circledR}$ therapy programs are effective in increasing the vocal intensity during structured speech tasks and improving self-perception of voice among individuals with PD [12].

Watts reported the outcomes of SPEAK OUT! ${ }^{\circledR}$ program is based on a retrospective data analysis of 78 participants with PD [1]. These participants were monitored for mean intensity during different structured speech tasks, including sustained vowels, reading, and conversation at the end of 12 sessions of the SPEAK OUT! ${ }^{\circledR}$ program, at 6-month follow-up, and 12-month follow-up. Results indicated a significant treatment effect on mean intensity values across all three speech tasks post-treatment and up to one year after treatment compared to the pre-treatment baseline. Large effect sizes $\left(\eta^{2}\right.$ values ranging from .685 to .793) were reported when baseline levels were compared to post-treatment and two consecutive follow-up periods. The author recommended additional studies examining the effect of SPEAK OUT! ${ }^{\circledR}$ and LOUD Crowd ${ }^{\circledR}$ program in study design with both treatment and control groups help dissociate the impact of treatment-related factors.

More recently, Boutsen and his colleagues reported the effectiveness of SPEAK OUT! $!^{\circledR}$ and LOUD Crowd ${ }^{\circledR}$ on the pro- sodic performance of individuals with PD [5]. Results indicated a significant improvement in speech intensity, pitch range, pitch variability, sustained vowel duration, vocal quality, reading intelligibility, and self-reported QOL performance before and after completion of the program. The study also reported a link between deterioration in speech performance and increased disease severity. Most recently, Behrman and her colleagues (2020) reported treatment data specific to vocal intensity, prosody, vocal quality, and self-reported VRQOL based on SPEAK OUT! ${ }^{\circledR}$ and LOUD Crowd ${ }^{\circledR}$ program [13]. The study included a treatment group of 47 participants with PD and a control group of 35 neurologically healthy adults. The disease severity of the PD participants ranged from mild to moderate (range $=1-3$ ) based on the Hoehn \& Yahr (H \& Y) scale. All participants completed a reading task and a monologue task. Participants with PD were recorded at baseline, last session of the SPEAK OUT! ${ }^{\circledR}$ program, and during two sessions (five weeks apart) of the LOUD Crowd ${ }^{\circledR}$ program. The control group participants were also recorded during a similar time frame. Results indicated statistically significant changes in mean intensity and frequency variability for both reading and monologue among participants with PD following treatment. In addition, participants with PD demonstrated significantly higher values of the cepstral peak prominence (CPP) and VRQOL scores post-therapy, thereby indicating improvements in overall voice quality and voice-related quality of life, respectively, following treatment.

One of the interesting findings of the study was that participants with PD demonstrated similar mean intensity and CPP compared to the control group after therapy. However, the participants with PD demonstrated lower values for all outcome variables than the control group. In contrast, the control group had no statistically significant change for any of the outcome variables across time for the monologue from baseline to post-therapy datapoints. The authors concluded that the improvements in the PD group scores were likely due to the effects of the SPEAK OUT! ${ }^{\circledR}$ and the LOUD Crowd ${ }^{\circledR}$ program. The study did not include any formal cognitive screenings for the enrolled participants. Therefore, the authors recommended future studies with formal, standardized assessments of cognitive abilities and long-term studies to better understand the efficacy of the SPEAK OUT! ${ }^{\circledR}$ and the LOUD Crowd ${ }^{\circledR}$ programs.

In conclusion, the limited existing studies on SPEAK OUT! $!^{\circledR}$ and LOUD Crowd ${ }^{\circledR}$ suggest improvements in vocal intensity, vocal quality, reading intelligibility, and VRQOL of individuals 
with PD. However, none of the prior studies included a comparison of treatment and control groups consisting of individuals with PD across multiple speech and non-speech domains such as cognitive status, swallowing abilities, and self-reported depression. In addition, almost all studies except the study by Watts have reported the treatment outcomes for relatively shorter periods ranging from few weeks to as long as four months following completion of the SPEAK OUT! ${ }^{\circledR}$ program.

Based on the existing evidence and the need to identify the best possible speech-based treatment outcomes for individuals with PD, the current study was designed to examine the effectiveness of the LOUD Crowd ${ }^{\circledR}$ program for six months. The research question was: Are there group differences in terms of speech, swallowing, cognitive performance, self-reported depression, and VRQOL of individuals with PD following a 6-month LOUD Crowd ${ }^{\circledR}$ program? Based on the available evidence, we hypothesized that the treatment (TX) group would demonstrate benefits across all domains compared to the control $(\mathrm{CN})$ group at the end of the six months.

\section{METHODS}

\section{Participants}

The current study was approved by the Institutional Review Board (AS-19-21-STW) prior to data collection. Participants included seven individuals with PD (4 males, 3 females) in the TX group (Mean age $=71.83 \mathrm{yr}$ ) and three individuals with PD ( 2 males, 1 female) in the $\mathrm{CN}$ group (Mean age $=75.67 \mathrm{yr}$ ). The TX group included two with mild, three with mild-moderate, and two moderate severity of hypokinetic dysarthria. In contrast, the $\mathrm{CN}$ participants included two with mild-moderate and one with moderate severity of hypokinetic dysarthria. Some of the study participants' speech characteristics included monoloudness, harsh and breathy voice, fast rate of speech, and imprecise articulation. Participants in the TX group had previously received intervention by completing the SPEAK OUT! ${ }^{\circledR}$ program and attended weekly sessions of LOUD Crowd $^{\circledR}$ at the time of data collection.

In contrast, the $\mathrm{CN}$ group had no prior or current speech intervention for their PD-related symptoms. One participant in the TX group withdrew from the study at the end of 3 months due to a stroke and subsequent hospitalization for multiple weeks. Therefore, data from six participants in the TX group and three participants in the $\mathrm{CN}$ group were analyzed for group comparisons. Table 1 includes the demographic information of the TX and $\mathrm{CN}$ groups.

\section{Procedures}

All participants completed a case history during the first visit. For each of the six months, all participants completed speech

Table 1. Demographic Information and Summary of Measures for the Participants with PD

\begin{tabular}{|c|c|c|}
\hline Domains & TX Group & CN Group \\
\hline Number of participants & 6 (4 males, 2 females) & 3 (2 males, 1 female) \\
\hline \multicolumn{3}{|l|}{ Hypokinetic severity } \\
\hline Mild & 2 & 0 \\
\hline Mild-moderate & 2 & 2 \\
\hline Moderate & 2 & 1 \\
\hline \multicolumn{3}{|l|}{ Hoehn \& Yahr Scale Score } \\
\hline Pre-test & $2.08 \pm 0.49$ & $2.08 \pm 0.49$ \\
\hline Post-test & $2.17 \pm 0.29$ & $2.33 \pm 0.58$ \\
\hline \multicolumn{3}{|c|}{ Dementia Rating Scale (DRS-2) } \\
\hline Pre-test & $134.4 \pm 4.04$ & $137.2 \pm 6.69$ \\
\hline Post-test & $136 \pm 4.58$ & $133 \pm 4.0$ \\
\hline \multicolumn{3}{|c|}{ Swallowing Disturbance Questionnaire (SDQ) } \\
\hline Pre-test & $6.17 \pm 4.62$ & $8 \pm 2.90$ \\
\hline Post-test & $5.67 \pm 5.03$ & $15 \pm 12.42$ \\
\hline \multicolumn{3}{|l|}{ Voice Handicap Index (VHI) } \\
\hline Pre-test & $11.17 \pm 5.50$ & $9.50 \pm 5.50$ \\
\hline Post-test & $11.67 \pm 4.17$ & $13.33 \pm 7.21$ \\
\hline
\end{tabular}

$\mathrm{TX}$, treatment group; $\mathrm{CN}$, control group. 
recordings (including vowels, glides, reading, and monologue) for six sessions. The monthly sessions were usually completed during the first two weeks of each month for all participants. All sessions were completed during self-reported ON medication states of participants. All recordings were completed in a quiet office/lab space. In addition to monthly voice recordings, all participants completed the Dementia Rating Scale (DRS-2) [14], the Swallowing Disturbance Questionnaire (SDQ) [15], and the Voice Handicap Index (VHI) [16] twice, once at baseline, and again at the end of six months.

\section{Acoustic analysis}

Acoustic analyses for the sustained vowel phonation tasks were completed using Praat [17]. The mean F0 was defined as the average vocal fold vibration frequency of a speaker. Based on the visual analysis of the F0 trace, pitch range settings were adjusted, and a range of $50-150 \mathrm{~Hz}$ was chosen for male speakers with $\mathrm{PD}$, and a range of $150-300 \mathrm{~Hz}$ was chosen for female speakers with PD. A voice report was generated using these settings to determine the mean F0, standard deviation (SD) of F0, jitter, shimmer, mean harmonic to noise ratio (HNR) for the sustained vowel phonation tasks completed by the participants. Specific to formants (F1 and F2), the formant range was set to $0-5,000 \mathrm{~Hz}$ with a window length of $25 \mathrm{~ms}$ and a dynamic range of $30 \mathrm{~dB}$. Formants were plotted in $\mathrm{Hz}$ using the phonR package [18] in R [19]. Each of the three vowels' (/a/, /i/, /u/) formants (F1, F2) were measured at midpoint using the linear predictive coding (LPC) function with default

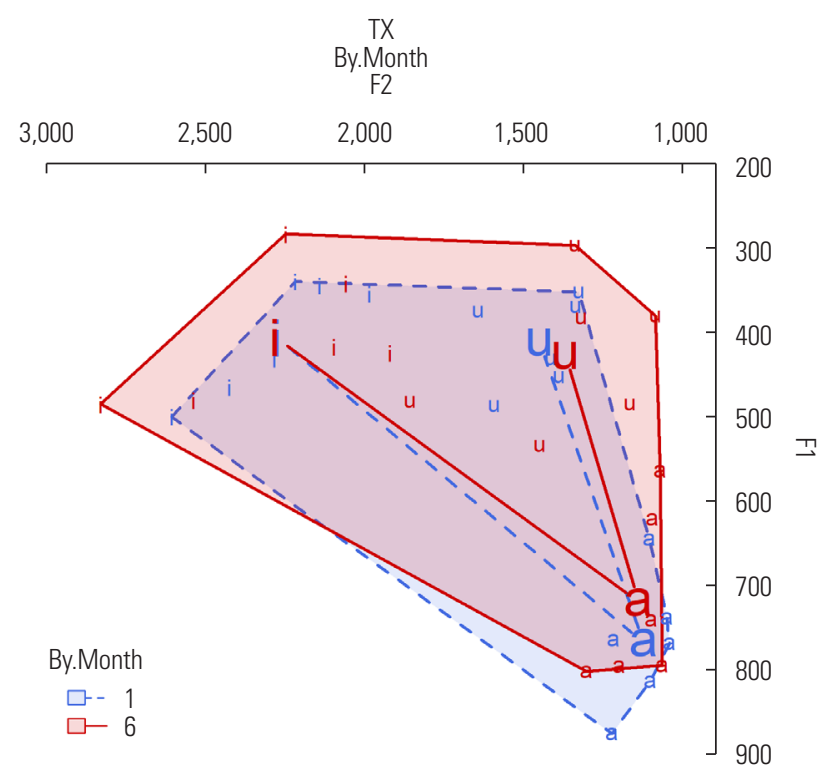

Figure 1. Vowel space of TX group. settings in Praat [17].

\section{Statistical analysis}

The current study included a small sample size of six participants with PD in the TX group and three participants with PD in the CN group. The independent variable was the group with two levels (TX and CN). The dependent variables included test scores on the DRS-2, H \& Y Scale, SDQ, and the VHI. Due to the small sample size and non-normality of the data distribution, nonparametric test of Mann-Whitney U test was completed to determine differences between the two participants groups for the different dependent variables. Normality tests were completed using SPSS Version 26. However, the dataset did not meet the normality assumption.

\section{RESULTS}

Due to the small sample size and failure to meet normality test assumptions, nonparametric tests were completed to determine the group differences. Independent Samples MannWhitney U-tests were completed for both groups for all of the measures, including H \& Y scores, DRS-2 scores, SDQ scores, and VHI scores. Results indicated no significant group differences between the two groups (TX and $\mathrm{CN}$ ) for baseline and at the end of 6 months $(p>0.05)$. Although no statistical group differences were noted, the group's detailed examination means suggested interesting trends between the two groups, which are summarized in the following sections.

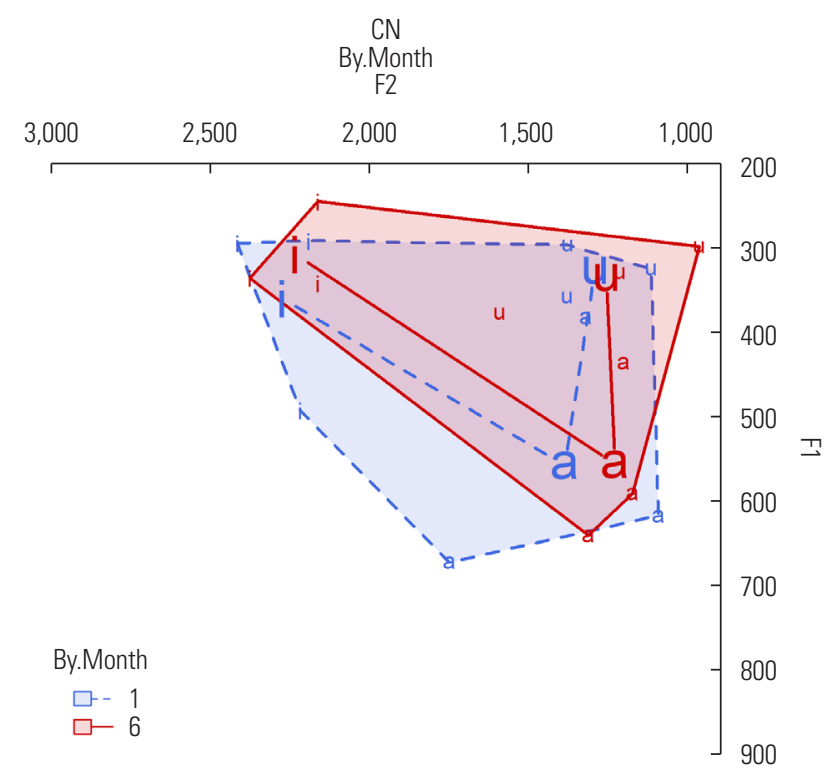

Figure 2. Vowel space of CN group. 


\section{Vowel space and vocal loudness}

Acoustic analyses revealed that the TX group's vowel space was found to be larger at the end of 6-months when compared to the corresponding values by the $\mathrm{CN}$ group. Figures 1 and 2 represent the baseline and 6-month values for the vowel space area for the TX and CN groups, respectively. In addition, Figure 3 represents the vowel space area of all of the speakers from both TX and CN groups. Next, Table 2 includes the live vocal intensity during different speech tasks (including sustained phonation, reading, and monologue). The live vocal intensity was tracked as it is often one of the most frequently reported changes associated with PD. The parameter of vocal intensity can provide indirect information about the phonatory dynamics. The live loudness values for the TX group were higher for most speech stimuli at baseline and all the stimuli at the end of the six months compared to the corresponding values of the $\mathrm{CN}$ group. Typically, an increase of 6 $\mathrm{dB}$ represents a double level of sound pressure level. In the current study, the relative difference between baseline and 6-month live loudness levels for the TX group changed by 7 $\mathrm{dBHL}$ or more during both reading (Rainbow passage and Grandfather passage) and monologue as compared to only a $7 \mathrm{dBHL}$ change for Rainbow passage in the $\mathrm{CN}$ group. In addi- tion to mean pitch and live speech intensity, other acoustic measurements including the jitter, shimmer, mean harmonic to noise ratio, and voice breaks were calculated for the vowels produced by the participants at baseline and at the end of six months. Table 3 includes a summary of all voice-related mea-

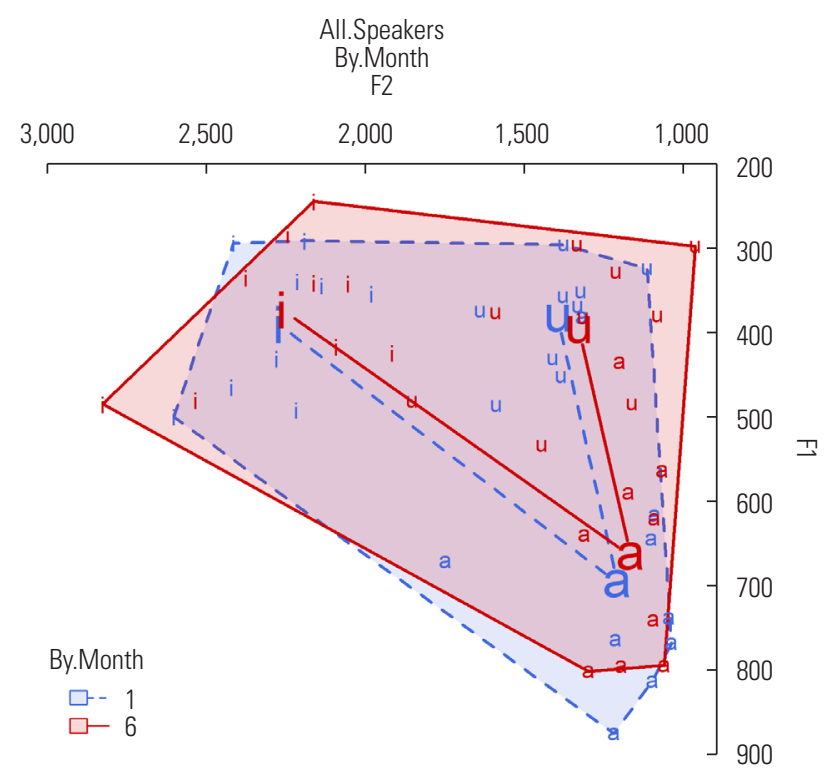

Figure 3. Vowel space of all speakers.

Table 2. Vocal Intensity Changes Over Time (Unit: dB SPL)

\begin{tabular}{|c|c|c|c|c|c|c|c|c|c|c|c|c|c|}
\hline \multirow{2}{*}{ Speech stimuli } & \multirow[b]{2}{*}{ Measures } & \multicolumn{2}{|c|}{ Month 1} & \multicolumn{2}{|c|}{ Month 2} & \multicolumn{2}{|c|}{ Month 3} & \multicolumn{2}{|c|}{ Month 4} & \multicolumn{2}{|c|}{ Month 5} & \multicolumn{2}{|c|}{ Month 6} \\
\hline & & $\mathrm{TX}$ & $\mathrm{CN}$ & $\mathrm{TX}$ & $\mathrm{CN}$ & $\mathrm{TX}$ & $\mathrm{CN}$ & $\mathrm{TX}$ & $\mathrm{CN}$ & $\mathrm{TX}$ & $\mathrm{CN}$ & $\mathrm{TX}$ & $\mathrm{CN}$ \\
\hline \multirow[t]{2}{*}{ Sustained /a/ } & $M$ & 85.75 & 74.83 & 85.25 & 71.78 & 82.90 & 72.80 & 82.66 & 74.52 & 85.57 & 82.68 & 88.11 & 78.27 \\
\hline & SD & 3.18 & 6.53 & 3.87 & 3.69 & 2.27 & 5.89 & 3.70 & 5.30 & 3.26 & 5.55 & 2.75 & 3.13 \\
\hline \multirow[t]{2}{*}{ Sustained /i/ } & M & 80.88 & 72.42 & 82.92 & 73.27 & 79.13 & 73.10 & 80.36 & 74.35 & 82.66 & 80.86 & 84.64 & 80.45 \\
\hline & SD & 3.42 & 4.96 & 4.92 & 4.24 & 2.21 & 1.90 & 5.90 & 6.19 & 5.57 & 5.13 & 3.40 & 2.61 \\
\hline \multirow[t]{2}{*}{ Sustained /u/ } & $\mathrm{M}$ & 84.88 & 74.0 & 85.08 & 73.01 & 80.62 & 74.35 & 79.71 & 77.91 & 83.74 & 82.61 & 86.57 & 80.45 \\
\hline & SD & 2.87 & 6.06 & 5.06 & 3.12 & 4.64 & 5.06 & 5.53 & 9.00 & 7.98 & 6.86 & 5.12 & 3.03 \\
\hline \multirow[t]{2}{*}{ Glide /a/ Minimum } & M & 79.92 & 58.50 & 79.50 & 79.73 & 74.60 & 73.83 & 74.58 & 75.03 & 76.32 & 84.43 & 81.77 & 78.17 \\
\hline & SD & 5.57 & 14.85 & 6.44 & 2.83 & 10.01 & 1.15 & 4.03 & 5.00 & 6.67 & 6.30 & 5.93 & 7.87 \\
\hline \multirow[t]{2}{*}{ Glide /a/ Maximum } & M & 87.42 & 88.50 & 93.83 & 85.80 & 89.78 & 86.67 & 89.98 & 87.17 & 92.28 & 89.63 & 95.25 & 89.93 \\
\hline & SD & 8.60 & 1.50 & 3.13 & 4.66 & 4.03 & 5.00 & 6.37 & 3.71 & 5.13 & 7.34 & 5.72 & 3.46 \\
\hline \multirow[t]{2}{*}{ Reading (Rainbow) } & $M$ & 72.58 & 69.17 & 75.84 & 74.38 & 77.51 & 71.76 & 79.01 & 73.70 & 77.03 & 78.13 & 80.83 & 76.79 \\
\hline & SD & 3.41 & 4.17 & 4.01 & 4.70 & 2.06 & 3.30 & 6.37 & 2.20 & 4.05 & 2.02 & 3.78 & 5.67 \\
\hline \multirow[t]{2}{*}{ Reading (Grandfather) } & M & 72.92 & 72.99 & 76.00 & 77.23 & 78.88 & 71.65 & 76.54 & 70.06 & 76.71 & 78.91 & 80.35 & 76.08 \\
\hline & $\mathrm{SD}$ & 3.01 & 5.67 & 4.27 & 7.18 & 1.77 & 2.72 & 4.03 & 3.42 & 4.51 & 1.38 & 5.69 & 4.55 \\
\hline \multirow[t]{2}{*}{ Monologue } & M & 69.25 & 72.75 & 72.73 & 71.42 & 73.74 & 68.27 & 70.41 & 70.56 & 73.02 & 77.29 & 77.52 & 73.78 \\
\hline & SD & 5.13 & 1.52 & 4.74 & 1.46 & 3.79 & 5.76 & 4.44 & 4.51 & 6.54 & 1.86 & 3.59 & 7.92 \\
\hline
\end{tabular}

$\mathrm{TX}$, treatment group; $\mathrm{CN}$, control group. 
surements related to the vowel tasks by the study participants.

\section{Swallowing and cognitive outcomes}

The average SDQ scores at baseline were 6.17 and 5.67 for the TX and CN groups, respectively. However, the 6-months scores for the two groups were markedly different, with an av- erage score of 8 for the TX group and a corresponding score of 15 for the CN group. The scores are suggestive of more difficulties in swallowing among the $\mathrm{CN}$ group when compared to the TX group at the end of 6-months. Specific to cognitive outcomes, the average DRS-2 scores at baseline for the TX and $\mathrm{CN}$ group were 134.4 and 136, respectively. However, the

Table 3. Summary of acoustic measurements of vowel productions completed by male (M) and female (F) participants with PD in the treatment (TX) group and the control (CN) group during the first (1st) and sixth (6th) month

\begin{tabular}{|c|c|c|c|c|c|c|c|c|c|c|c|c|c|c|}
\hline & \multicolumn{2}{|c|}{ Mean F0 } & \multicolumn{2}{|c|}{ F0 SD } & \multicolumn{2}{|c|}{ MPT } & \multicolumn{2}{|c|}{ Jitter } & \multicolumn{2}{|c|}{ Shimmer } & \multicolumn{2}{|c|}{$\begin{array}{l}\text { Voice } \\
\text { breaks }\end{array}$} & \multicolumn{2}{|c|}{ Mean HNR } \\
\hline & $1 \mathrm{st}$ & 6th & $1 \mathrm{st}$ & 6th & $1 \mathrm{st}$ & 6th & $1 \mathrm{st}$ & 6th & $1 \mathrm{st}$ & 6th & 1st & 6th & $1 \mathrm{st}$ & 6th \\
\hline \multicolumn{15}{|l|}{ Vowel /a/ } \\
\hline TX_M_01 & 95.129 & 123.639 & 23.308 & 19.794 & 9.819 & 9.808 & .741 & .689 & 7.094 & 8.254 & 1 & 1 & 13.939 & 14.745 \\
\hline TX_F_02 & 193.208 & 215.179 & 2.946 & 3.746 & 14.941 & 18.504 & .786 & .183 & 2.827 & 1.194 & 0 & 0 & 19.366 & 28.404 \\
\hline TX_M_03 & 110.677 & 109.731 & 4.686 & 19.759 & 18.194 & 8.181 & .232 & .478 & 3.023 & 2.173 & 0 & 1 & 18.813 & 20.626 \\
\hline TX_F_04 & 230.33 & 238.53 & 4.312 & 4.193 & 8.942 & 7.724 & .27 & .334 & 3.903 & 3.31 & 1 & 0 & 22.358 & 23.654 \\
\hline TX_M_06 & 161.749 & 132.656 & 7.587 & 22.981 & 22.874 & 20.586 & .192 & .293 & 1.362 & 1.9 & 0 & 0 & 24.642 & 25.063 \\
\hline TX_M_07 & 158.844 & 113.84 & 13.291 & 13.8 & 12.743 & 22.925 & 1.105 & .566 & 5.528 & 4.943 & 0 & 0 & 13.603 & 16.146 \\
\hline CN_F_01 & 250.402 & 245.299 & 17.928 & 12.484 & 34.259 & 26.459 & 2.173 & 1.689 & 9.933 & 7.248 & 16 & 7 & 15.525 & 19.484 \\
\hline CN_M_02 & 156.225 & 154.015 & 3.874 & 4.816 & 5.131 & 10.248 & .477 & .21 & 2.046 & 2.097 & 0 & 0 & 26.28 & 17.594 \\
\hline CN_M_03 & 168.749 & 181.616 & 4.066 & 3.126 & 5.713 & 7.52 & .617 & .335 & 4.13 & 2.096 & 0 & 0 & 17.861 & 24.844 \\
\hline \multicolumn{15}{|l|}{ Vowel/i/ } \\
\hline TX_M_01 & 62.322 & 156.677 & 4.865 & 3.687 & 14.226 & 7.876 & .642 & .28 & 7.695 & 1.875 & 0 & 1 & 10.211 & 21.932 \\
\hline TX_F_02 & 120.07 & 224.044 & 1.487 & 2.41 & 20.09 & 19.973 & .174 & .338 & 1.662 & 2.372 & 0 & 0 & 24.006 & 22.657 \\
\hline TX_M_03 & 109.361 & 113.501 & 6.324 & 5.53 & 15.938 & 10.911 & .242 & .482 & 3.316 & 1.918 & 0 & 1 & 15.56 & 9.317 \\
\hline TX_F_04 & 252.784 & 230.448 & 5.48 & 5.628 & 10.301 & 9.317 & .353 & .397 & 11.503 & 3.183 & 0 & 0 & 12.591 & 23.44 \\
\hline TX_M_06 & 165.446 & 141.774 & 18.376 & 3.341 & 19.627 & 24.798 & .213 & .21 & .814 & .886 & 0 & 0 & 31.399 & 31.367 \\
\hline TX_M_07 & 164.626 & 81.163 & 7.174 & 27.243 & 10.983 & 26.656 & .469 & .552 & 2.912 & 2.858 & 1 & 0 & 19.247 & 11.058 \\
\hline CN_F_01 & 253.212 & 252.456 & 13.462 & 20.299 & 41.461 & 21.379 & 2.283 & 5.197 & 6.096 & 12.552 & 21 & 32 & 16.699 & 12.618 \\
\hline CN_M_02 & 159.021 & 153.775 & 2.686 & 2.854 & 5.982 & 7.329 & .302 & .195 & 1.598 & 1.532 & 0 & 0 & 30.121 & 18.047 \\
\hline CN_M_03 & 166.436 & 169.487 & 8.356 & 3.598 & 8.835 & 12.51 & .551 & .477 & 1.745 & 1.405 & 1 & 0 & 24.436 & 27.673 \\
\hline \multicolumn{15}{|l|}{ Vowel /u/ } \\
\hline TX_M_01 & 75.231 & 155.9 & 21.556 & 4.399 & 10.688 & 8.27 & .63 & .41 & 5.241 & 2.22 & 1 & 0 & 18.607 & 25.479 \\
\hline TX_F_02 & 120.418 & 229.108 & 1.66 & 3.223 & 21.668 & 18.24 & .202 & .168 & 1.815 & 2.057 & 0 & 1 & 27.671 & 26.872 \\
\hline TX_M_03 & 107.552 & 110.724 & 8.326 & 7.728 & 8.472 & 8.624 & .425 & .331 & 2.298 & 1.248 & 0 & 0 & 22.005 & 27.124 \\
\hline TX_F_04 & 250.526 & 253.436 & 8.7088 & 5.175 & 8.033 & 9.11 & .326 & 1.61 & 1.643 & 7.267 & 1 & 1 & 22.009 & 21.104 \\
\hline TX_M_06 & 170.943 & 145.498 & 2.118 & 3.016 & 23.565 & 23.573 & .625 & .157 & .11 & .696 & 0 & 0 & 36.207 & 35.089 \\
\hline TX_M_07 & 173.41 & 109.688 & 3.383 & 25.558 & 11.802 & 17.68 & .733 & .911 & 4.848 & 4.674 & 0 & 0 & 20.135 & 15.064 \\
\hline CN_F_01 & 280.894 & 254.886 & 20.829 & 24.839 & 20.711 & 20.055 & .297 & 4.24 & 2.53 & 7.134 & 7 & 36 & 30.646 & 18.717 \\
\hline CN_M_O2 & 155.903 & 153.802 & 2.342 & 2.054 & 9.206 & 9.529 & .289 & .145 & 1.928 & 2.529 & 0 & 0 & 30.247 & 27.544 \\
\hline CN_M_03 & 166.822 & 170.521 & 3.448 & 6.691 & 8.771 & 9.693 & .541 & .481 & 1.766 & 1.711 & 0 & 0 & 24.575 & 29.364 \\
\hline
\end{tabular}

Mean FO, FO SD are in Hz; Maximum Phonation Time (MPT) in seconds. 
Table 4. Summary of different domains and total scores of Voice Handicap Index (VHI)

\begin{tabular}{|c|c|c|c|c|c|c|c|c|}
\hline & Pre_F & Post_F & Pre_P & Post_P & Pre_E & Post_E & Pre_Total & Post_Tota \\
\hline TX_M_01 & 11 & 13 & 14 & 16 & 10 & 13 & 35 & 42 \\
\hline TX_F_02 & 5 & 12 & 8 & 10 & 3 & 8 & 16 & 30 \\
\hline TX_M_03 & 20 & 18 & 11 & 14 & 21 & 17 & 52 & 44 \\
\hline TX_F_04 & 14 & 12 & 8 & 7 & 9 & 9 & 31 & 28 \\
\hline TX_M_06 & 6 & 5 & 7 & 8 & 12 & 5 & 25 & 18 \\
\hline TX_M_07 & 12 & 11 & 19 & 16 & 12 & 5 & 43 & 32 \\
\hline CN_F_01 & 5 & 7 & 12 & 15 & 3 & 12 & 20 & 34 \\
\hline CN_M_O2 & 11 & 17 & 12 & 12 & 16 & 11 & 39 & 40 \\
\hline CN_M_03 & 16 & 21 & 18 & 16 & 16 & 17 & 50 & 54 \\
\hline
\end{tabular}

F, Functional; P, Physical; E, Emotional; Pre, baseline scores; Post, scores at the end of 6 months.

average DRS-2 scores at the end of 6-months for both groups were 137.2 and 133, respectively. Specifically, four out of six participants in the TX group demonstrated improved scores on DRS-2 at the end of 6-months. In contrast, only one out of 3 participants in the $\mathrm{CN}$ group demonstrated improved DRS-2 scores at the end of 6-months. Table 1 includes the mean and standard deviation values of SDQ and DRS-2 scores for TX and $\mathrm{CN}$ groups.

\section{Self-reported depression and voice related quality of life outcomes}

The mean baseline BDI-II scores for the TX and CN groups were 12.6 and 7, respectively. However, at the end of 6-months, the mean for the CN group doubled (i.e., 14.3) while the TX group mean was 12 points. Four out of six participants in the TX self-reported had similar or improved scores at the end of 6-months. However, all three participants in the CN group selfreported higher scores (i.e., higher self-reported depression) at the end of 6-months. Specific to VRQOL performance, participants in the TX group reported relatively better VHI scores with a mean of 32.33 at the end of 6-months when compared to a baseline score of 33.67. In contrast, the corresponding 6 -month and baselines VHI values for the CN group were 42.67 and 36.33, respectively. Therefore, participants in the TX group self-reported improvements in VRQOL at the end of six months compared to participants in the $\mathrm{CN}$ group. Table 4 includes scores of subdomains and total VHI for each participant.

\section{DISCUSSION}

The current study examined the benefits of the LOUD Crowd ${ }^{\circledR}$ program on speech, voice, cognition, swallowing, self-reported depression, and VRQOL of participants with PD. To the best of our knowledge, the current study is one of the few available studies that has reported outcomes from a LOUD Crowd ${ }^{\circledR}$ program on multiple aspects of performance among participants with PD during six months. Specific to vocal loudness performance, both TX and CN groups demonstrated an increase in live loudness for all speech tasks at the end of six months. However, the overall difference in live loudness levels was relatively more visible for the TX group for both reading and monologue tasks when compared to the $\mathrm{CN}$ group. The findings of improved live loudness levels in the TX group are similar to prior studies $[5,10,11]$.

Results also suggested the presence of a larger vowel space for the TX group compared to the CN group. Speakers with large vowel spaces tend to represent more wide dispersed vowel productions and thus often considered to be more intelligible during speech tasks [20,21]. Although the current study did not specifically measure the speakers' speech intelligibility during different points, the pre and post vowel space area of the TX group indicates possible benefits of the groupbased LOUD Crowd ${ }^{\circledR}$ therapy. Specifically, the TX group has a larger vowel space at the end of the six months, suggesting clearer articulation than the $\mathrm{CN}$ group.

Participants in the TX group demonstrated better cognitive, swallowing, self-reported depression, and VRQOL outcomes at the end of 6-months when compared to the CN group. These findings are similar to prior study findings $[4,10]$. The positive experience of swallowing could be attributed to increasing laryngeal sphincteric activity based on the weekly intervention and other compensatory strategies, leading to the more effective protection of the airway among the TX group 
participants. This is the first LOUD Crowd ${ }^{\circledR}$ study with both treatment and control groups monitored for multiple domains (including speech, cognition, swallowing, and VRQOL). The findings suggest possible positive benefits of the LOUD Crowd ${ }^{\circledR}$ program. Some of the current study limitations included smaller participant groups, a homogenous group of participants from similar geographical backgrounds, the unequal sample size for the TX and the CN group, and the lack of clients in more advanced PD stages, thereby leading to a limited generalization of the current study findings. Future studies with larger sample sizes, participants with different severities of PD, and comparisons between the LOUD Crowd ${ }^{\circledR}$ program and LSVT ${ }^{\circledR}$ program can further help establish evidence for effective treatment programs for individuals with PD.

\section{ACKNOWLEDGMENTS}

The author would like to thank all of the participants with PD for their participation as well as Ms. Kay Headrick and Ms. Nancy Payne, for their assistance with data collection. In addition, the author would like to acknowledge the assistance of Dr. Valerie Freeman with vowel space area related figures. Portions of the current study were presented at the 2nd Pan American Parkinson's Disease and Movement Disorders Congress at Miami (FL, USA) in 2018.

\section{STATEMENT OF ETHICS}

The Humans Subject Review Board approved the study at the author's institution, and all participants provided written consent.

\section{FINANCIAL/NON-FINANCIAL DISCLOSURE AND CONFLICTS OF INTEREST}

None to report.

\section{FUNDING SOURCE}

The current study received no specific grant from any funding agency in the public, commercial, or not-for-profit sectors.

\section{AUTHOR CONTRIBUTIONS}

The author designed the study and wrote the manuscript.

\section{REFERENCES}

1. Watts CR. A retrospective study of long term intensity in hypokinetic dysarthria. BMC Ear, Nose and Throat Disorders. 2016;16: DOI 10.1186/s12901-016-0022-8.

2. Parkinson's foundation. Retrieved on June 28, 2020, from https:// www.parkinson.org/Understanding-Parkinsons/Statistics

3. Miller N. Speech, voice and language in Parkinson's disease: changes and interventions. Neurodegenerative Disease Management. 2012;2:279-289.

4. De Angelis EC, Mourao LF, Ferraz HB, Behlau MS, Pontes PAL, Andrade LAF. Effect of voice rehabilitation on oral communication of Parkinson's disease patients. Acta Neurologica Scandinavica. 1997;96:199-205.

5. Boutsen F, Park E, Dvorak J, Cid C. Prosodic improvement in persons with Parkinson disease receiving SPEAK OUT! ${ }^{\circledR}$ voice therapy. Folia Phoniatrica Logopedica. 2018;70:51-58.

6. Wiley K, Elandary S. SPEAK OUT! ${ }^{\circledR}$ a practical approach to treating Parkinson's. San Antonio: Texas Speech and Hearing Association Annual Convention; 2014.

7. Spielman J, Ramig LO, Mahler L, Halpern A, Gavin WJ. Effects of an extended version of the Lee Silverman voice treatment on voice and speech in Parkinson's disease. American Journal of Speech Language Pathology. 2017;16:95-107.

8. Searl J, Wilson K, Haring K, Dietsch AM, Lyons K. Feasibility of group voice therapy for individuals with Parkinson's disease. Special Education and Communication Disorders Faculty Publications. 2011;44:719-732.

9. Edwards A, Theodoros D, Davidson B. Group therapy for maintenance of speech in Parkinson's disease following LSVT LOUD: a pilot study. Speech, Language and Hearing. 2018;21:105-116.

10. Levitt J. A case study: the effects of the "SPEAK OUT! ${ }^{\circledR 1 "}$ voice program for Parkinson's disease. International Journal of Applied Science and Technology. 2014;4:20-28.

11. Cleveland L, Pimentel J, Talarico M, Solomon J. Vocal intensity and speech intelligibility pre- and post- SPEAK OUT! therapy. Presented at the Annual Convention of American Speech-Language-Pathology; 2015.

12. Levitt J, Chitnis S, Walker-Batson D. The effects of the "SPEAK OUT! ${ }^{\circledR "}$ and "LOUD Crowd ${ }^{\circledR "}$ programs for Parkinson disease. International Journal of Health Sciences. 2015;3:13-19.

13. Behrmann A, Cody J, Elandary S, Flom P, Chitnis S. The effect of SPEAK OUT! and the LOUD Crowd on dysarthria due to Parkinson's disease. American Journal of Speech-Language Pathology. 2020; doi: https://doi.org/10.1044/2020_AJSLP-19-00024.

14. Mattis S. The Dementia Rating Scale-2 (DRS-2). 2004. Lutz, FL: Psychological Assessment Resources.

15. Cohen JT, Manor Y. Swallowing disturbance questionnaire for detecting dysphagia. The Laryngoscope. 2011; 121: 1383-1387.

16. Jenkinson C, Fitzpatrick R, Peto V. Health-related quality-of-life measurement in patients with Parkinson's disease. Pharmacoeconomics. 1999;15:157-165.

17. Boersma P, Weenink D. (2020). Praat: Doing phonetics by comput- 
er, version 6.1.16. http://www.praat.org/.

18. McCloy D. (2016). phonR: tools for phoneticians and phonologists. $R$ package version 1.0-7. https://CRAN.R-project.org/package=phonR.

19. R Core Team (2020). R: A language and environment for statistical computing, version 4.0.2. R Foundation for Statistical Computing, Vienna, Austria. https://www.R-project.org/.
20. Neel AT. Vowel space characteristics and vowel identification accuracy. Journal of Speech Language and Hearing. 2008;51:574585.

21. Bond ZS, Moore TJ. A note on the acoustic-phonetic characteristics of inadvertently clear speech. Speech Communication. 1994; 14:325-337. 